\title{
Autologous bone graft versus PEKK cage for vertebral replacement after 1- or 2-level anterior median corpectomy
}

\author{
Stefan Koehler, MD, ${ }^{1}$ Furat Raslan, MD, ${ }^{2}$ Christian Stetter, MD, ${ }^{1}$ Stefan Mark Rueckriegel, MD, ${ }^{1}$ \\ Ralf-Ingo Ernestus, MD, ${ }^{1}$ and Thomas Westermaier, MD'1 \\ 1Department of Neurosurgery, University Hospital Wuerzburg; and '2Department of Neurosurgery, Klinikum Hohe Warte, \\ Bayreuth, Germany
}

\begin{abstract}
OBJECTIVE Anterior cervical corpectomy with fusion has become the most widely used procedure for the treatment of multilevel cervical stenosis. Although an autologous bone graft is the gold standard for vertebral replacement after corpectomy, industrial implants have become popular because they result in no donor-site morbidity. In this study, the authors compared clinical and radiological results of autologous iliac grafts versus those of bone-filled polyetherketoneketone (PEKK) cage implants.
\end{abstract}

METHODS The clinical and radiological data of 46 patients with degenerative multilevel cervical stenosis and who underwent 1- or 2-level anterior median corpectomy between 2004 and 2012 were analyzed. The patients in Group 1 were treated with vertebral replacement with an autologous iliac graft, and those in Group 2 were treated with a PEKK cage implant. Each patient also underwent osteosynthesis with an anterior plate-screw system. Visual analog scale (VAS) and European Myelopathy Scale scores, loss of height and regional cervical lordosis angle, and complication rates of the 2 groups were compared.

RESULTS The mean follow-up time was 20 months. In both groups, the VAS and European Myelopathy Scale scores improved significantly. The loss of height was $3.7 \%$ in patients with iliac grafts and $5.3 \%$ in patients with PEKK implants. The rates of osseous fusion were similar in Groups 1 and 2 (94.7\% and $91.3 \%$, respectively). At the end of the follow-up period, none of the patients complained about donor-site pain. One patient in Group 1 suffered a fracture of the iliac bone that required osteosynthesis. Four patients in Group 2 had to receive revision surgery for cage and/or plate-screw dislocation and new neurological deficit or intractable pain.

CONCLUSIONS Preoperative pain and radicular and myelopathic symptoms improve after decompression irrespective of the material used for vertebral replacement. The use of PEKK cages for vertebral replacement seems to result in a higher risk of implant-related complications. A prospective randomized study is necessary to supply evidence for the use of autografts and artificial implants after anterior cervical corpectomy with fusion.

http://thejns.org/doi/abs/10.3171/2015.5.SPINE14887

KEY WORDS corpectomy; ACCF; autologous bone graft; PEEK; PEKK; cage; cervical

A NTERIOR cervical corpectomy followed by anterior fusion (ACCF) has become a widely used procedure for the treatment of multilevel degenerative cervical stenosis. After the pioneering work of Smith and Robinson $^{12}$ on an anterior approach to the cervical spine, removal of the intervertebral disc, and interbody fusion in 1958, the implantation of an autologous bone graft, most frequently taken from the iliac crest, combined with an anterior plate-screw osteosynthesis remained the gold standard of vertebral replacement and has been reported to result in fusion rates of 70\%-100\%, .,13 In recent years, numerous nonbiological implants have been developed and introduced for clinical use to avoid donor-site morbidities such as additional pain, delayed mobilization, and iliac fracture. ${ }^{1,10}$ Titanium cages are the most widely used xenografts for use in ACCF, and several groups have reported

ABBREVIATIONS ACCF = anterior cervical corpectomy with fusion; EMS = European Myelopathy Scale; PEKK = polyetherketoneketone; VAS = visual analog scale SUBMITTED Augst 27, 2014. ACCEPTED May 12, 2015.

INCLUDE WHEN CITING Published online October 16, 2015; DOI: 10.3171/2015.5.SPINE14887. 
good clinical and radiological results with them, ${ }^{14-16}$ but significant numbers of implant-related complications, such as implant failure and subsidence, have been mentioned. ${ }^{4,5}$ Polyetherketone cages have the theoretical advantage of being less rigid than titanium cages and, therefore, might result in reduced cage subsidence and a lower rate of dislocation.

To the best of our knowledge, there has been no systematic analysis to directly compare the clinical and radiological courses after ACCF using autologous bone grafts or polyetherketoneketone (PEKK) cages. It was the aim of this study to analyze the clinical and radiological outcomes of patients with autologous bone graft or a PEKK cage for vertebral replacement after ACCF of the cervical spine.

\section{Methods \\ Patient Collective}

Between 2004 and 2012, 137 patients underwent a 1- or 2-level ACCF in the Department of Neurosurgery, University Hospital Wuerzburg. Forty patients were treated for trauma, infection, or tumor, 12 received an implant other than a PEKK cage, and 15 simultaneously underwent additional posterior instrumentation and were excluded from further investigation. Of the remaining 70 patients, $24 \mathrm{did}$ not continue follow-up for the minimum of 12 months and were excluded from further analysis. The clinical and radiological data of the remaining 46 patients (mean age 67 \pm 10 years) with degenerative multilevel cervical stenosis who underwent a 1- or 2-level ACCF between 2004 and 2012 were analyzed retrospectively. In our department, PEKK cages (Invadur-Oxpekk cage, PINA Medizintechnik Vertriebs AG) were introduced for vertebral replacement in 2007. Each patient in whom surgery was performed between 2004 and 2007 underwent vertebral replacement with an autologous iliac graft (Group 1, $\mathrm{n}=$ 19), and those who underwent surgery between 2007 and 2012 received a PEKK cage (Group 2, $n=27$ ). All of the patients also underwent osteosynthesis with an anterior plate-screw system (cervical spine locking plate [CSLP] system, Synthes). Decompression in both groups was performed in an identical way. ACCF was performed using a common anterior approach via a right-sided skin incision. Foraminal decompression was performed bilaterally with excision of the posterior longitudinal ligament to ensure adequate neural decompression. In Group 1, a large tricortical bone graft was harvested from the right iliac crest and fit into the vertebrectomy space. In Group 2, the PEKK cages were filled with spongious bone obtained from the osseus decompression.

All patients had radiological signs of a multilevel degenerative spinal stenosis with clinical and electrophysiological (somatosensory and motor evoked potentials) signs of cervical myelopathy and/or radiculopathy.

\section{Complications}

Surgical complications were recorded to differentiate implant-related and general complications. The numbers of surgical revisions in both groups were compared.

\section{Radiological Assessment}

For each patient, preoperative CT scans, MRI scans, and digitalized lateral and anteroposterior radiographs of the cervical spine were obtained. A digital lateral radiograph or CT scan of every patient was obtained on Day 1 after surgery. Radiographs were taken periodically until at least 12 months after the operation. A CT scan of the cervical spine was obtained at the end of follow-up. For this purpose, a thin-slice (1.5-mm) CT scan (SOMATOM 4+ Volume Zoom CT Scanner, Siemens Healthcare Diagnostics $\mathrm{GmbH}$ ), which included the disc space above and below the fused vertebrae, and triplanar reconstructions were performed. Measurements of the height of the spondylodesis made from sagittal reconstructions of the CT images and from lateral radiographs on Day 1 after surgery and at the end of follow-up were compared and used for the radiological follow-up.

The loss of height during the follow-up period was determined by comparing the total height of the spondylodesis between the first postoperative image on Day 1 after surgery and that at the end of follow-up. The total height of the spondylodesis was measured from the superior endplate of the cranial vertebra to the inferior endplate of the caudal vertebra (Fig. 1). Digital measurements for use in our analyses were made by using the Picture Archiving and Communication System (syngo.plaza PACS, Siemens).

The relative height of the implant was determined by measuring the height of the bone graft or cage and divid-

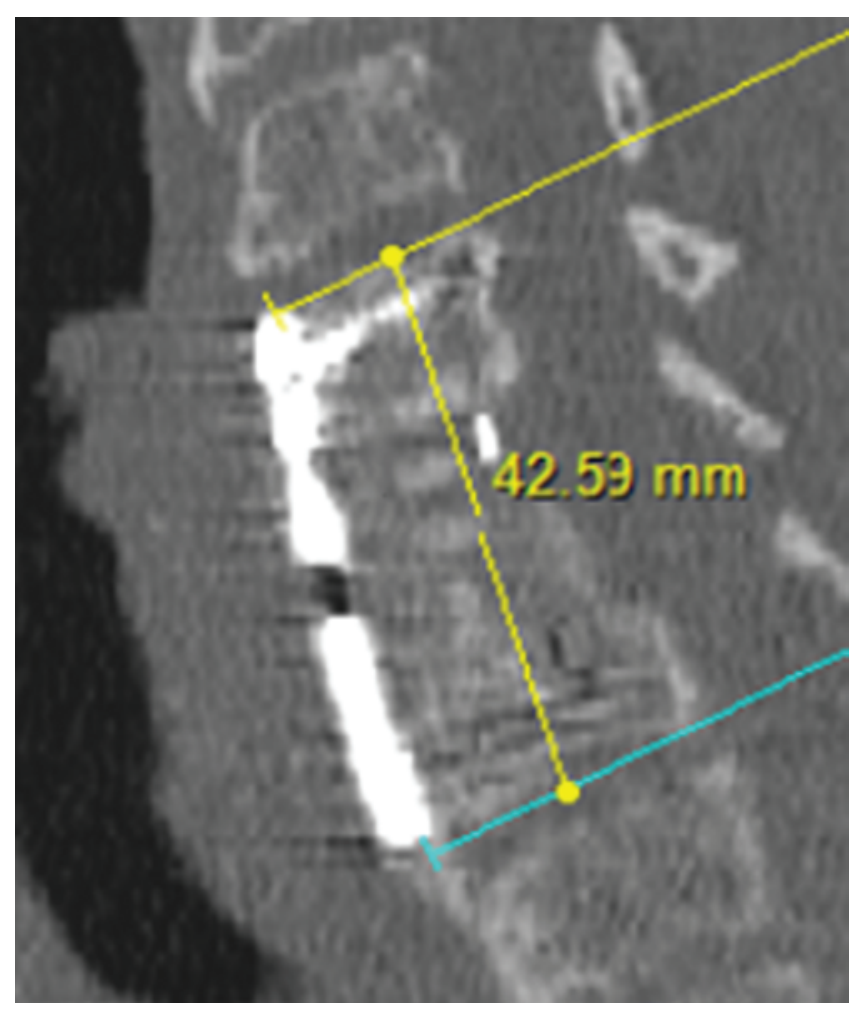

FIG. 1. The total height of the spondylodesis was measured as the distance from the superior endplate of the cranial vertebra to the inferior endplate of the caudal vertebra. For these measurements, digital Picture Archiving and Communication System (PACS) tools were used. Figure is available in color online only. 
ing it by the total height of the spondylodesis to obtain information about the extent and manner of graft subsidence in the 2 groups.

The local cervical lordosis angle over the fused vertebrae was measured on Day 1 after surgery and at the end of follow-up. If there was a kyphotic alignment of the vertebrae, the angles were defined as negative.

The fusion rates in both groups were determined. Osseous fusion was considered if a bony bridge without interruption was found in the images at the end of follow-up.

\section{Clinical Assessment}

All patients underwent standardized neurological assessments preoperatively and periodically postoperatively until the end of follow-up. Pain levels were assessed by using visual analog scale (VAS) scores, and functional outcomes were classified by using European Myelopathy Scale (EMS) scores.

\section{Statistical Analysis}

Results are presented as means \pm SD. Normally distributed parametric data were compared by using the Student t-test to compare the 2 groups. Incidences were compared by using the Fisher exact test. Statistical analysis was performed by using GraphPad Prism statistical software. A $p$ value of $<0.05$ was considered statistically significant.

\section{Results \\ Patient Collective}

Forty-six patients met the inclusion criteria. Twentyeight patients were male, and 18 were female. Nineteen patients underwent vertebral replacement with an autologous iliac crest bone graft (Group 1), and 27 were treated with a PEKK-cage implant (Group 2). The mean ages at the time of surgery were $66 \pm 11$ years [Group 1] and $67 \pm$ 10 years [Group 2]. The mean follow-up times were $19.2 \pm$ 15.2 months [Group 1] and $21.9 \pm 13.6$ months [Group 2]. In Group 1, 14 (73.7\%) patients underwent a 1-level ACCF, and 5 (26.3\%) patients underwent a 2-level ACCF. In Group 2, 19 (70.4\%) patients underwent a 1-level ACCF, and $8(29.6 \%)$ patients underwent a 2-level ACCF.

\section{General Complications}

Significant rebleeding into the cervical soft tissue, leading to early revision surgery, occurred in 2 (4.3\%) patients, 1 in each group. One patient in Group 2 required early revision surgery as a result of CSF leakage. None of the patients had a persistent new neurological deficit at the end of follow-up.

\section{Implant-Related Complications}

One patient in Group 1 experienced a fracture of the iliac crest that required surgical revision by an osteosynthesis of the iliac bone. No other implant-related complications occurred in this group. In particular, there was no dislocation of the bone graft or the anterior plate-screw system.

In Group 2, a minor dislocation of the plate-screw system in 1 patient was recorded, and in another patient, a minor loosening of the screws occurred, but revision surgery was not needed. In 4 patients of Group 2, a dislocation of the PEKK cage and loosening of the plate-screw system with a new neurological deficit and/or intractable pain made revision surgery necessary. In 2 of these patients, anterior revision of the PEKK cage and plate-screw system was performed after 3 and 4 months, respectively. In another patient, a 2-level corpectomy had to be extended to a 3-level corpectomy with replacement of the PEKK cage by an autologous bone graft and subsequent posterior instrumentation 1 week after the first operation. In the fourth case, an anterior revision procedure followed by posterior instrumentation was performed because of persistent pain and failed bony fusion (Table 1).

\section{Radiological Assessment}

Patients who underwent cervical revision surgery and/ or secondary posterior instrumentation were excluded from further radiological assessment. Thus, 19 patients in Group 1 and 23 patients in Group 2 were investigated.

In Group 1, the mean total height of the spondylodesis was $48.0 \pm 9.0 \mathrm{~mm}$ on Day 1 after surgery and decreased to $46.3 \pm 9.1 \mathrm{~mm}(96.3 \% \pm 4.1 \%)$ at the end of follow-up. In Group 2, the total height of the spondylodesis was $51.7 \pm$ $9.7 \mathrm{~mm}$ on Day 1 after surgery and decreased to $48.9 \pm 8.8$ $\mathrm{mm}(94.7 \% \pm 3.6 \%)$ at the end of follow-up (Fig. 2). The differences did not reach the level of significance $(\mathrm{p}=0.17$ for loss of height during follow-up).

In Group 1, the mean ratio between the height of the cage and the height of the fused vertebrae on Day 1 after surgery was $0.48 \pm 0.09$; at the end of follow-up, the mean ratio decreased to $0.47 \pm 0.09$. In Group 2, the mean ratio was $0.52 \pm 0.07$ on Day 1 and increased to $0.54 \pm 0.06$ at the end of follow-up (Fig. 3).

In Group 1, the mean lordosis angle over the fused vertebrae decreased significantly $(\mathrm{p}<0.05)$ from $5.9^{\circ} \pm 5.2^{\circ}$ at Day 1 after surgery to $4.1^{\circ} \pm 4.4^{\circ}$ at the end of followup. In Group 2, the mean lordosis angle decreased significantly $(\mathrm{p}<0.05)$ from $6.6^{\circ} \pm 7.0^{\circ}$ to $3.5^{\circ} \pm 6.0^{\circ}$. The differences between the groups were not statistically significant.

Osseous fusion at the end of follow-up was achieved in $18(94.7 \%)$ of 19 patients in Group 1 and in 21 (91.3\%) of 23 patients in Group 2. The difference between the groups was not statistically significant.

TABLE 1. Number of surgical complications that required revision surgery after ACCF*

\begin{tabular}{lccc}
\hline \multicolumn{1}{c}{ Complication } & $\begin{array}{c}\text { Group 1 (iliac } \\
\text { crest autograft, } \\
\mathrm{n}=19)\end{array}$ & $\begin{array}{c}\text { Group 2 } \\
(\text { PEKK cage, } \\
\mathrm{n}=27)\end{array}$ & $\begin{array}{c}\mathrm{p} \\
\text { Value }\end{array}$ \\
\hline Postoperative bleeding & 1 & 1 & \\
\hline CSF fistula & 0 & 1 & \\
\hline Infection & 0 & 0 & \\
\hline $\begin{array}{l}\text { Implant-related complication } \\
\text { (donor site) }\end{array}$ & 1 & 0 & \\
\hline $\begin{array}{l}\text { Implant-related complication } \\
\text { (implant site) }\end{array}$ & 0 & 4 & 0.28 \\
\hline Total & 2 & 6 & 0.46 \\
\hline
\end{tabular}

* Differences between the 2 groups were not statistically significant. 


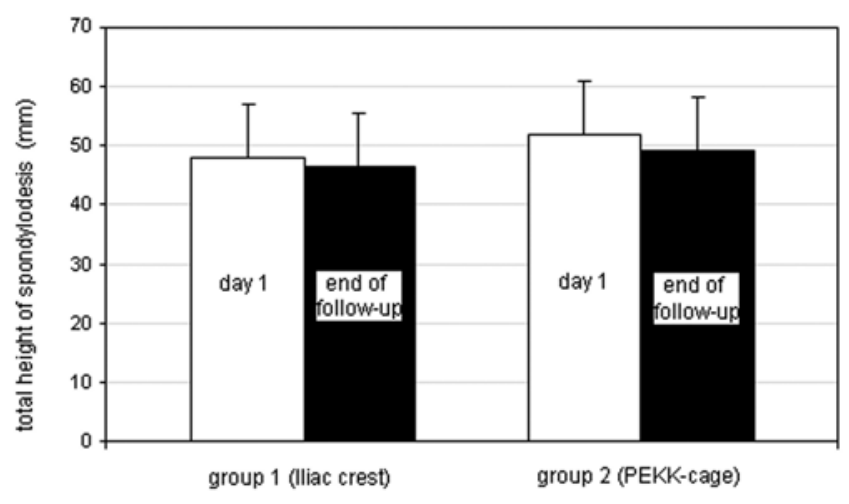

FIG. 2. The mean losses of height were $3.6 \%$ in Group 1 and $5.3 \%$ in Group 2. The difference between the 2 groups did not reach the level of significance $(p=0.17)$.

\section{Clinical Assessment}

The mean reported pain VAS scores at the end of follow-up had improved significantly $(p<0.01)$ over those reported in the preoperative assessments in Group 1 (from $2.26 \pm 1.79$ to $1.11 \pm 0.99$ ) and in Group 2 (from $2.63 \pm$ 1.63 to $1.19 \pm 0.88$ ). The differences in the mean preoperative VAS scores and the mean improvement of pain according to the VAS scores (1.15 in Group 1 vs 1.44 in Group 2) were not statistically significant (Fig. 4 upper).

The EMS scores improved significantly $(\mathrm{p}<0.001)$ from $14.74 \pm 1.56$ preoperatively to $16.05 \pm 1.18$ at the end of follow-up in Group 1 and from $14.15 \pm 2.49$ to $15.63 \pm$ 1.94 in Group 2. The differences in the mean preoperative EMS scores and those at the end of follow-up and the mean improvement of EMS scores (1.31 [Group 1] vs 1.48 [Group 2]) were not statistically significant between the 2 groups (Fig. 4 lower).

\section{Discussion}

In this study, we retrospectively analyzed 46 patients who underwent a 1- or 2-level ACCF performed to treat

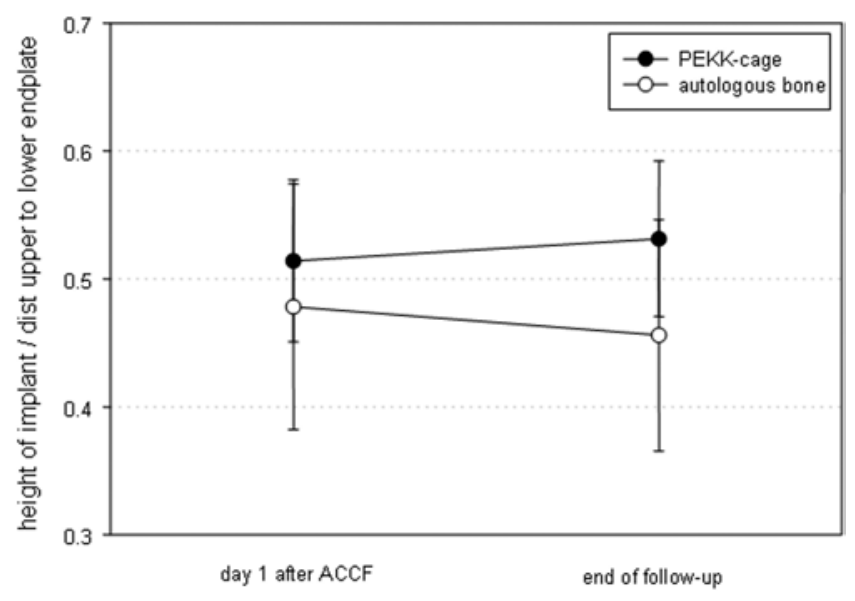

FIG. 3. The mean loss of height in Group 1 was caused by impaction of the bony implant, as depicted by the mean decrease of the relative height of the implant related to the total height of the spondylodesis. In Group 2, the mean relative height of the implants increased, which suggests that the implants impressed the adjacent vertebrae and their endplates.
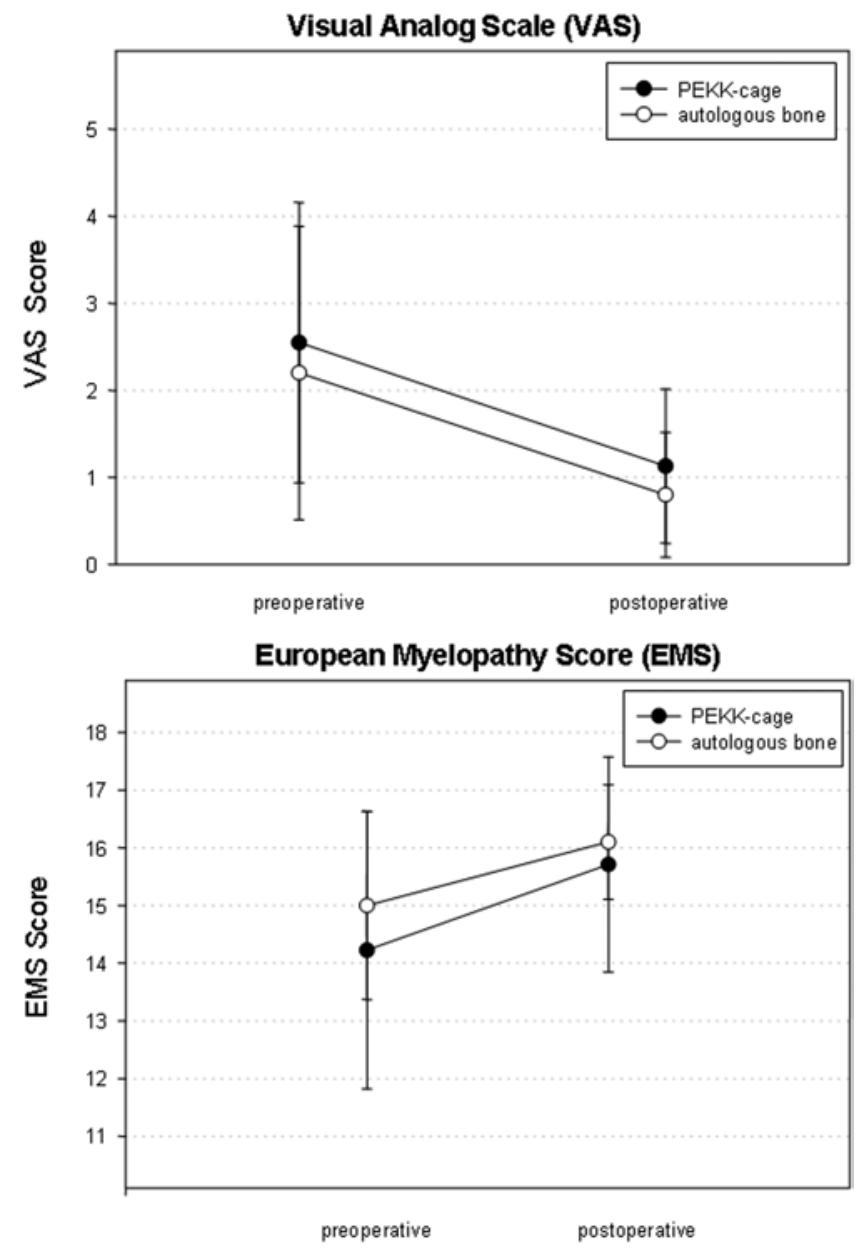

FIG. 4. In both groups, preoperative pain and signs of cervical myelopathy, as determined by VAS (upper) and EMS (lower) scores, showed long-term decreases.

degenerative disorders of the cervical spine. Nineteen patients underwent vertebral replacement with an autologous iliac crest bone graft, and 27 patients were treated by using a PEKK cage. Until 2007, we exclusively used bone grafts for vertebral replacement in our department for this group of patients. After 2007, only PEKK cages were used in the treatment of degenerative diseases of the cervical spine. Because there were no criteria for the choice of the implant other than the year in which the patient underwent surgery, this retrospective analysis has the characteristics of a quasi-randomized trial; this is confirmed by the patient data, which are nearly identical in both groups concerning age, sex, number of fused vertebrae, and preoperative radiological and clinical presentations.

To our knowledge, there have been no previous reports of studies in the literature that assessed the fusion and complication rates of PEKK cages used for ACCF. Titanium grafts are the most widely used implants for this purpose and have been shown to result in fusion rates of $70 \%$ $100 \% .{ }^{14-16}$ In some of these reports, however, a remarkable rate of implant-related complications were mentioned. ${ }^{2}$ In our series, general complications occurred in both groups at comparable frequencies. This result is plausible because CSF leaks and hematomas are complications that are in- 
dependent of the fusion materials used. However, there was an obvious difference in the incidence of implantrelated complications between the 2 groups. There was 1 patient with significant donor-site morbidity (fracture of the iliac crest) that required iliac osteosynthesis. Avoiding donor-site morbidity is the most striking argument for the use of artificial implants, ${ }^{1,7}$ and this case in our series also illustrates the problem. Donor-site morbidity may include pain, hematoma, fracture, or meralgia paresthetica and has been reported to occur in up to $25 \%$ of patients. Some authors reported that most of these complaints resolved over time, and others observed that significant pain at the donor site could persist for more than 24 months after surgery or that chronic pain and functional impairment could persist even longer after vertebral replacement by an iliac crest autograft. $1,10,11$ With the exception of this single case, we were unable to reproduce these results; no patient in our study reported long-term problems concerning the donor site at the end of follow-up period.

Concerning the local implant failure that necessitated revision surgery, our study showed a considerable accumulation of these cases in the PEKK cage group. There was no dislocation of an iliac crest bone graft or adjacent osteosynthetic material noted, which is in contrast to 4 cases of dislocation of the PEKK cage and adjacent plate-screw system in the cage group. This difference is remarkable and suggests that the fusion was more stable with the use of the bone graft. However, our finding that the relative height of the implant in relation to the total height of the spondylodesis decreased in the bone-graft group and increased in the cage group suggests different mechanisms of cage impaction. In the bone-graft group, the loss of height was caused by impaction of the bone graft itself, and in the cage group, the loss of height was caused by impaction of the rigid cage into the adjacent endplates, which may explain the greater loss of height in the cage group. Once the endplate-the strongest, weight-loading part of the vertebral body - is weakened, the resistance decreases and the construction might become more unstable and prone to lose height. ${ }^{9}$ Thus, this pathomechanism might be determined to be the reason for the loosening of screws and the breakout of implants. An implant-related strain on the endplates is a well known phenomenon after anterior cervical discectomy with fusion (ACDF) involving polyetheretherketone (PEEK) cages or polymethylmethacrylate (PMMA) substitutes. ${ }^{6,8}$ After ACDF, however, this problem is obviously less eminent because of the shorter distance and the lower degree of instability caused by the decompressive procedure than those in ACCF procedures. A certain degree of impaction of the implant is normal and probably promotes osseous fusion. This is underlined by the postoperative reduction of the local lordosis angle and by the fact that the loss of height in the patients who did not undergo revision surgery had no correlation with the clinical outcomes measured by VAS and EMS scores. The clinical results were good, and there was a significant improvement of both parameters and an improvement in all patients.

\section{Conclusions}

In this series, the rate of implant failures that necessi- tated revision surgery was higher in the group of patients who had undergone a PEKK cage implant for spondylodesis than in the group of patients who had undergone ACCF with an autologous bone graft. The radiological data suggest that intrusion of the cage into the adjacent vertebrae might contribute to a higher rate of implant and screwplate dislocation. The drawbacks of this analysis are that the data originated from a relatively small number of patients and, although the setting was quasi-randomized, from a retrospective analysis. A prospective randomized trial to compare the clinical and radiological outcomes after vertebral body replacement with autologous bone grafts and those after various artificial implants are used instead could provide more evidence for or against their use in ACCF.

\section{References}

1. Banwart JC, Asher MA, Hassanein RS: Iliac crest bone graft harvest donor site morbidity. A statistical evaluation. Spine (Phila Pa 1976) 20:1055-1060, 1995

2. Boakye M, Patil CG, Ho C, Lad SP: Cervical corpectomy: complications and outcomes. Neurosurgery 63 (4 Suppl 2):295-302, 2008

3. Brown JA, Havel P, Ebraheim N, Greenblatt SH, Jackson WT: Cervical stabilization by plate and bone fusion. Spine (Phila Pa 1976) 13:236-240, 1988

4. Castellvi AE, Castellvi A, Clabeaux DH: Corpectomy with titanium cage reconstruction in the cervical spine. J Clin Neurosci 19:517-521, 2012

5. Daubs MD: Early failures following cervical corpectomy reconstruction with titanium mesh cages and anterior plating. Spine (Phila Pa 1976) 30:1402-1406, 2005

6. Gebremariam L, Koes BW, Peul WC, Huisstede BM: Evaluation of treatment effectiveness for the herniated cervical disc: a systematic review. Spine (Phila Pa 1976) 37:E109-E118, 2012

7. Kim MK, Kim SM, Jeon KM, Kim TS: Radiographic comparison of four anterior fusion methods in two level cervical disc diseases: autograft plate fixation versus cage plate fixation versus stand-alone cage fusion versus corpectomy and plate fixation. J Korean Neurosurg Soc 51:135-140, 2012

8. Park JI, Cho DC, Kim KT, Sung JK: Anterior cervical discectomy and fusion using a stand-alone polyetheretherketone cage packed with local autobone: assessment of bone fusion and subsidence. J Korean Neurosurg Soc 54:189-193, 2013

9. Porto Filho MR, Pastorello MT, Defino HL: Experimental study of the participation of the vertebral endplate in the integration of bone grafts. Eur Spine J 14:965-970, 2005

10. Sawin PD, Traynelis VC, Menezes AH: A comparative analysis of fusion rates and donor-site morbidity for autogeneic rib and iliac crest bone grafts in posterior cervical fusions. $\mathbf{J}$ Neurosurg 88:255-265, 1998

11. Silber JS, Anderson DG, Daffner SD, Brislin BT, Leland JM, Hilibrand AS, et al: Donor site morbidity after anterior iliac crest bone harvest for single-level anterior cervical discectomy and fusion. Spine (Phila Pa 1976) 28:134-139, 2003

12. Smith GW, Robinson RA: The treatment of certain cervicalspine disorders by anterior removal of the intervertebral disc and interbody fusion. J Bone Joint Surg Am 40-A:607-624, 1958

13. Swank ML, Lowery GL, Bhat AL, McDonough RF: Anterior cervical allograft arthrodesis and instrumentation: multilevel interbody grafting or strut graft reconstruction. Eur Spine J 6:138-143, 1997

14. Uribe JS, Sangala JR, Duckworth EA, Vale FL: Comparison between anterior cervical discectomy fusion and cervical 
corpectomy fusion using titanium cages for reconstruction: analysis of outcome and long-term follow-up. Eur Spine J 18:654-662, 2009

15. Waschke A, Kaczor S, Walter J, Duenisch P, Kalff R, Ewald $\mathrm{C}$ : Expandable titanium cages for anterior column cervical reconstruction and their effect on sagittal profile: a review of 48 cases. Acta Neurochir (Wien) 155:801-807, 2013

16. Woiciechowsky C: Distractable vertebral cages for reconstruction after cervical corpectomy. Spine (Phila Pa 1976) 30:1736-1741, 2005

\section{Disclosure}

Dr. Westermaier received payments from Johnson \& Johnson for teaching activities (spinal neurosurgery).

\section{Author Contributions}

Conception and design: Koehler, Westermaier. Acquisition of data: Koehler, Raslan, Stetter, Westermaier. Analysis and interpretation of data: Koehler, Westermaier. Drafting the article: Koehler, Westermaier. Critically revising the article: Ernestus, Westermaier. Reviewed submitted version of manuscript: Raslan, Stetter, Rueckriegel, Ernestus, Westermaier. Approved the final version of the manuscript on behalf of all authors: Koehler. Statistical analysis: Westermaier. Administrative/technical/material support: Ernestus. Study supervision: Westermaier.

\section{Supplemental Information}

Current Affiliation

Dr. Raslan: Department of Neurosurgery, BG University Hospital Bergmannsheil, Bochum, Germany.

\section{Correspondence}

Stefan Koehler, Department of Neurosurgery, University Hospital Wuerzburg, Josef-Schneider-Strasse 11, Wuerzburg 97080, Germany. email: koehler_s1@ukw.de. 